\title{
Character expansion method for supergroups and extended superversions of the Leutwyler-Smilga and Berezin-Karpelevich integrals
}

\author{
C. Lehner and T. Wettig \\ Institute for Theoretical Physics, University of Regensburg, 93040 Regensburg, Germany \\ T. Guhr \\ Department of Physics, University of Duisburg-Essen, 47048 Duisburg, Germany \\ Y. Wei \\ School of Mathematical Sciences, University of Nottingham, Nottingham NGr2RD, UK
}

(Dated: May 9, 2008)

\begin{abstract}
We introduce an extension of the character expansion method to the case of supergroups. This method allows us to calculate a superversion of the Leutwyler-Smilga integral which, to the best of our knowledge, has not been calculated before. We also use the method to generalize a previously calculated superversion of the Berezin-Karpelevich integral. Our character expansion method should also allow for the calculation of other supergroup integrals.
\end{abstract}

\section{INTRODUCTION}

When solving models in field theory and statistical mechanics, one often faces the challenge to integrate over continuous groups or cosets. Examples can be found in a wide range of applications, comprising fields as different as condensed matter physics [1] and quantum gravity [2. There are also prominent applications in random matrix theory [3, 4, including statistical models in quantum chromodynamics [5]. From a mathematics viewpoint, group integration belongs to the field of harmonic analysis [6, 7]. Half a century ago, Hua [8 obtained invariant measures for a large class of spaces. Shortly thereafter, Harish-Chandra 9 derived his celebrated integration formula for Lie groups. The radial coordinates on which these integrals depend parametrize the space of the Cartan subalgebras. In the unitary case, Harish-Chandra's result coincides with the Itzykson-Zuber integral [10]. Unfortunately, HarishChandra's formula cannot be applied to the majority of integrals over orthogonal and unitary-symplectic groups that arise in physics, because these latter integrals depend on radial coordinates defining a space which is outside the group or its algebra, respectively. Hence they can be interpreted as certain matrix generalizations of Bessel functions, see a discussion in Ref. 11]. Group integrals related to but different from those mentioned so far are of high relevance for applications in quantum chromodynamics. Particularly important are integrals of the Berezin-Karpelevich [12, 13, 14] and Leutwyler-Smilga [15] type. Although in these cases the integration is over unitary groups, Harish-Chandra's formula cannot be used either, because the integrands are of a different form.

All these considerations carry over to integrals over supergroups. Supermathematics [16] — in the present context often referred to as supersymmetry — was introduced to the theory of disordered systems by Efetov [17] and subsequently to random matrix theory by Verbaarschot, Weidenmüller, and Zirnbauer [18, 19. Supersymmetry is nowadays an indispensable tool for many applications, once more including those in quantum chromodynamics [1, 4, 5].

At present, there are three different methods for exact calculations of the integrals discussed above: (1) The diffusion equation method was developed by Itzykson and Zuber [10 and in Refs. 13, 14 for ordinary space. The ItzyksonZuber integral was generalized to supermathematics 20 by a proper extension of the diffusion equation method which was then further extended to work out the supersymmetric Berezin-Karpelevich integral [13, 21]. In Ref. 222, the diffusion equation method was generalized beyond the unitary case to prove the supersymmetric Harish-Chandra formula that had been conjectured in Refs. [23, 24]. This also provided a new proof for the ordinary Harish-Chandra formula. (2) Balantekin introduced the character expansion method for integrals over unitary groups in ordinary space [25, 26]. The method was further extended in Ref. [27]. Even some integrals over ordinary orthogonal and unitary-symplectic groups could be calculated using a generalization of the character expansion method 28. (3) Shatashvili [29] used an explicit parametrization of the unitary group in terms of Gelfand-Tzetlin coordinates [30] to calculate correlation functions in the Itzykson-Zuber model. This method was extended to supersymmetry in Ref. [31] and considerably generalized to obtain recursive solutions for a wide class of radial functions which include group integrals as special cases [11, 32].

The main focus of the present contribution is an extension of the character expansion method to supergroups. This extension then allows us to calculate a supersymmetric version of the Leutwyler-Smilga integral. The ordinary version of this integral yields the finite-volume partition function of quantum chromodynamics in the so-called epsilon-regime [15, 33, and its superversion has applications in related ("partially quenched") theories that contain both fermionic and bosonic degrees of freedom. We also generalize the result previously obtained in Ref. [13, for a 
supersymmetric Berezin-Karpelevich integral.

This article is organized as follows. In Sec. II] the integrals in question are defined and the results of our calculation are given. A general outline of our character expansion method is presented in Sec. III. We then apply the method to the calculation of the supersymmetric Leutwyler-Smilga and Berezin-Karpelevich integrals in Sec. IV] We summarize our results and give an outlook to further applications of the supersymmetric character expansion method in Sec. $\mathrm{V}$. Three appendices are provided to collect various algebraic theorems, to discuss a conjectured power series identity and its connection to Richardson-Littlewood coefficients, and to present explicit examples for the supersymmetric Leutwyler-Smilga integral.

\section{STATEMENT OF THE INTEGRALS}

To define the notation to be used in the subsequent sections, we start by stating the integrals that will be calculated using the character expansion methods introduced in Sec. III.

The supersymmetric Leutwyler-Smilga integral is defined as

$$
\mathcal{I}_{\mathrm{LS}} \equiv \int d \mu(U) \exp \left(\beta \operatorname{Str}\left(A U+B U^{-1}\right)\right)
$$

where $U \in \mathrm{U}(m \mid n), d \mu(U)$ is the invariant measure, $\beta \in \mathbb{C}$, and $A, B$ are arbitrary $(m+n) \times(m+n)$ supermatrices. We show that this integral is given by

$$
\mathcal{I}_{\mathrm{LS}}=\mathcal{C}_{m} \mathcal{C}_{n} \beta^{\frac{(m+n)-(m-n)^{2}}{2}} \frac{\operatorname{det}\left(\lambda_{j}^{m+n-i} I_{m+n-i}\left(2 \beta \lambda_{j}\right)\right)_{i, j=1, \ldots, m+n}}{\Delta\left(\lambda_{1}^{2}, \ldots, \lambda_{m}^{2}\right) \Delta\left(\lambda_{m+1}^{2}, \ldots, \lambda_{m+n}^{2}\right)}
$$

where $\lambda_{1}^{2}, \ldots, \lambda_{m+n}^{2}$ are the eigenvalues of the supermatrix $A B$ and $I_{\nu}$ is the modified Bessel function of the first kind. Furthermore, $\Delta\left(\lambda_{1}^{2}, \ldots, \lambda_{m}^{2}\right) \equiv \prod_{1 \leq i<j \leq m}\left(\lambda_{i}^{2}-\lambda_{j}^{2}\right)$ is the Vandermonde determinant and

$$
\mathcal{C}_{n} \equiv \prod_{k=1}^{n-1} k !
$$

Equation 22] correctly reproduces the result obtained in Ref. 27] for ordinary groups U $(m)$.

The second integral calculated in this work, the supersymmetric Berezin-Karpelevich integral, is defined as

$$
\mathcal{I}_{\mathrm{BK}} \equiv \int d \mu(U) \int d \mu(V) \exp \left(\beta \operatorname{Str}\left(U A V B+U^{-1} C V^{-1} D\right)\right),
$$

where $U, V \in \mathrm{U}(m \mid n), \beta \in \mathbb{C}$, and $A, B, C, D$ are arbitrary $(m+n) \times(m+n)$ supermatrices. We show that

$$
\mathcal{I}_{\mathrm{BK}}=\mathcal{C}_{m}^{2} \mathcal{C}_{n}^{2} \beta^{(m+n)-(m-n)^{2}} \frac{\operatorname{det}\left(I_{0}\left(2 \beta \lambda_{i} \mu_{j}\right)\right)_{i, j=1, \ldots, m} \operatorname{det}\left(I_{0}\left(2 \beta \lambda_{m+i} \mu_{m+j}\right)\right)_{i, j=1, \ldots, n}}{B\left(\lambda^{2} ; m, n\right) B\left(\mu^{2} ; m, n\right)}
$$

where $\lambda_{1}^{2}, \ldots, \lambda_{m+n}^{2}$ are the eigenvalues of the supermatrix $B C$ and $\mu_{1}^{2}, \ldots, \mu_{m+n}^{2}$ are the eigenvalues of the supermatrix $A D$. The Berezinian $B\left(\lambda^{2} ; m, n\right)$ is given by

$$
B\left(\lambda^{2} ; m, n\right)=\frac{\Delta\left(\lambda_{1}^{2}, \ldots, \lambda_{m}^{2}\right) \Delta\left(\lambda_{m+1}^{2}, \ldots, \lambda_{m+n}^{2}\right)}{\prod_{i=1}^{m} \prod_{j=1}^{n}\left(\lambda_{i}^{2}-\lambda_{m+j}^{2}\right)} .
$$

This extends the result obtained in Ref. [13] using the diffusion equation method.

Note that in the above results we have to assume that the supermatrices $A B$ (in case of $\mathcal{I}_{\mathrm{LS}}$ ) or $B C$ and $A D$ (in case of $\mathcal{I}_{\mathrm{BK}}$ ) are diagonalizable in the sense explained in App. A. However, our results can be extended to non-diagonalizable supermatrices using a limiting procedure which is discussed in App. A. Furthermore, the case of coinciding eigenvalues also requires a limiting procedure, which is discussed in App. B.

We prove our results in Secs. IV A and IVB. First, however, we discuss how the character expansion method can be extended to supersymmetric integrals in general. 


\section{CHARACTER EXPANSION FOR SUPERGROUPS}

The concept of the character expansion method for integrals over supergroups remains the same as for integrals over ordinary groups. We expand the integrand in terms of supergroup characters, use orthogonality relations of supermatrix representation elements in order to perform the integral, and identify the remaining power series.

We will consider integrals over a supermatrix $U \in \mathrm{U}(m \mid n)$. The integrands in question contain terms of the form

$$
\exp (\beta \operatorname{Str}(A U))=\sum_{n=0}^{\infty} \frac{\beta^{n}}{n !} \operatorname{Str}(A U)^{n}
$$

where $\beta \in \mathbb{C}$ and $A \in \operatorname{Gl}(m \mid n)$.

In order to expand the integrand in terms of supercharacters of $A U$ we make use of Balantekin's observation ${ }^{1}$ [34] that

$$
\operatorname{Str}(A U)^{n}=\sum_{t,|t|=n} \sigma_{t} \xi_{t}(A U)
$$

where the sum is over all Young diagrams $t$ with $n$ boxes that correspond to covariant representations ${ }^{2}$ and $\xi_{t}$ is the corresponding supercharacter. The expansion coefficient $\sigma_{t}$ of a Young diagram $t$ with $\hat{N}$ rows is given by 34

$$
\sigma_{t} \equiv|t| ! \frac{\Delta\left(\hat{k}_{1}, \ldots, \hat{k}_{\hat{N}}\right)}{\prod_{i=1}^{\hat{N}} \hat{k}_{i} !}
$$

where

$$
\hat{k}_{i} \equiv \hat{N}+t_{i}-i
$$

and $t_{i}$ is the number of boxes in the $i$-th row of the Young diagram. The character of a supergroup element in representation $t$ is defined as

$$
\xi_{t}(U) \equiv \operatorname{Str}\left(\Gamma^{t}(U)\right)=\sum_{i}(-1)^{\epsilon(i)} \Gamma_{i i}^{t}(U)
$$

with the supermatrix representation $\Gamma^{t}, \epsilon(i)=1$ if the index $i$ is fermionic and $\epsilon(i)=0$ otherwise. We are thus able to express the right-hand side of Eq. (8) in terms of the supermatrix representation elements $\Gamma_{a b}^{t}(U)$.

Note that our integrands always contain supertraces involving $U$ as well as $U^{-1}$ in the exponentials. With the help of the orthogonality relations of supermatrix representation elements [16],

$$
\int d \mu(U) \Gamma_{a b}^{r}(U) \Gamma_{c d}^{s}\left(U^{-1}\right)=\alpha_{r} \delta_{r s} \delta_{a d} \delta_{b c}(-1)^{\epsilon(c)}
$$

where $\alpha_{r}$ is the norm of the supergroup representation $r$, we are thus able to perform the integral over $U$. A careful treatment of signs is necessary due to the anticommutation of some supermatrix elements. For two supermatrices $A$ and $B$ this can be expressed by the formula

$$
A_{a b} B_{c d}=B_{c d} A_{a b}(-1)^{[\epsilon(a)+\epsilon(b)][\epsilon(c)+\epsilon(d)]} .
$$

We implicitly use the fact that the irreducible representations in the sum of Eq. (8) are constructed from their respective fundamental representation in the same way for $\mathrm{U}(m \mid n)$ and $\mathrm{Gl}(m \mid n)[35$.

After the integration over $U$ has been performed using Eq. (12), a power series corresponding to Eq. (8) remains. Because of the appearance of the factor $\alpha_{r}$ in Eq. (12), only non-degenerate covariant supergroup representations (i.e., those with $\alpha_{r} \neq 0$ ) contribute to this power series. In order to identify the power series with a known function we need explicit formulas for the supercharacter $\xi_{t}$ and the norm $\alpha_{t}$ of a given representation $t$. In 1981, Balantekin and Bars

\footnotetext{
${ }^{1}$ In Ref. 34 Balantekin only considered $\mathrm{U}(m \mid n)$, but his arguments also apply to $\operatorname{Gl}(m \mid n)$.

${ }^{2}$ I.e., representations that are constructed only from covariant bases by symmetrization of bases according to Young diagram $t$.
} 
obtained an integral formula for characters of supergroups [35, 36]. In the given form, however, it is not suited for an application to character expansion methods. In 1997, Alfaro, Medina, and Urrutia 37. obtained another formula for the supercharacters of a non-degenerate covariant supergroup representation, which is more useful in the present context. The restriction to non-degenerate representations does not pose any problem for the character expansion method because, as we have just seen, only these representations contribute to the result.

Alfaro, Medina, and Urrutia observed that any Young diagram $t$ describing a non-degenerate covariant representation consists of an $m \times n$ block in the top left corner and two sub-diagrams $p$ and $q^{T}$ such that $p$ and $q$ are legitimate Young diagrams of $\mathrm{Gl}(m)$ and $\mathrm{Gl}(n)$, respectively, cf. Fig. 1. The sub-diagram $p$ to the right of the $m \times n$ block is thus restricted to $m$ rows, while the sub-diagram $q$ that appears transposed below the $m \times n$ block is restricted to $n$ rows.

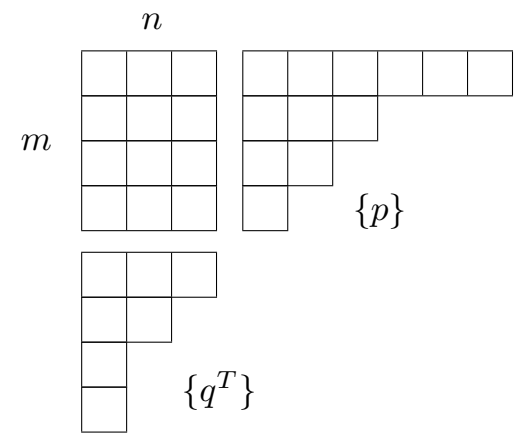

FIG. 1: Young diagram corresponding to a non-degenerate covariant representation of $\operatorname{Gl}(m \mid n)$.

The supercharacter of an $(m+n)$-dimensional supermatrix $A$ with eigenvalues $a_{1}, \ldots, a_{m+n}$ corresponding to $t$ is given by [37]

$$
\xi_{t}(A)=(-1)^{|q|} \Sigma(a ; m, n) \chi_{p}\left(a_{1}, \ldots, a_{m}\right) \chi_{q}\left(a_{m+1}, \ldots, a_{m+n}\right)
$$

where $|q|$ is the number of boxes in the sub-diagram $q$,

$$
\Sigma(a ; m, n) \equiv \prod_{i=1}^{m} \prod_{j=1}^{n}\left(a_{i}-a_{m+j}\right),
$$

and $\chi_{p}$ and $\chi_{q}$ are the characters of the ordinary groups $\operatorname{Gl}(m)$ and $\operatorname{Gl}(n)$ corresponding to the Young diagrams $p$ and $q$. Weyl's character formula [38] states

$$
\chi_{p}\left(a_{1}, \ldots, a_{m}\right)=\frac{\operatorname{det}\left(a_{i}^{k_{j}}\right)_{i, j=1, \ldots, m}}{\Delta\left(a_{1}, \ldots, a_{m}\right)}
$$

where

$$
k_{i} \equiv m+p_{i}-i
$$

for $1 \leq i \leq m$ and $p_{i}$ is the number of boxes in the $i$-th row of the Young diagram $p$.

The norm $\alpha_{t}$ of the representation shown in Fig. 1 depends on the sub-diagrams in the following manner [37,

$$
\alpha_{t}=(-1)^{|q|} \frac{|t| !}{|p| !|q| !} \frac{\sigma_{p} \sigma_{q}}{\sigma_{t}} \frac{1}{d_{p} d_{q}},
$$

where $|p|,|q|$, and $|t|$ count the boxes in the corresponding (sub-)diagram and $d_{p}$ and $d_{q}$ give the dimensions of the representations of $\mathrm{Gl}(m)$ and $\mathrm{Gl}(n)$ corresponding to Young diagrams $p$ and $q$, e.g.,

$$
d_{p}=\frac{\Delta\left(k_{1}, \ldots, k_{m}\right)}{\prod_{i=1}^{m}(m-i) !} .
$$


We can now replace the sum over all non-degenerate, covariant representations $t$ that remains from Eq. (8) after integration over $U$ by two sums over the sub-diagrams $p$ and $q$. An explicit way to write these sum is, e.g.,

$$
\sum_{k_{1}>k_{2}>\ldots>k_{m} \geq 0}
$$

for the sum over $p$ and

$$
\sum_{k_{m+1}>k_{m+2}>\ldots>k_{m+n} \geq 0}
$$

for the sum over $q$, where

$$
k_{i} \equiv m+n+q_{i-m}-i
$$

for $m<i \leq m+n$ and $q_{i-m}$ is the number of boxes in the $(i-m)$-th row of the Young diagram $q$.

This concludes the extension of the character expansion method to the case of supergroups. We now apply these considerations to the calculation of our supersymmetric integrals in Secs. IV A and IV B.

\section{CALCULATION OF THE INTEGRALS}

The supersymmetric extensions of the Leutwyler-Smilga and Berezin-Karpelevich integrals are calculated in Secs. IV A and IVB respectively.

\section{A. Supersymmetric Leutwyler-Smilga integral}

For convenience, we repeat the definition of the supersymmetric Leutwyler-Smilga integral given in Sec. II.

$$
\mathcal{I}_{\mathrm{LS}}=\int d \mu(U) \exp \left(\beta \operatorname{Str}\left(A U+B U^{-1}\right)\right)
$$

The notation is as in Sec. II. We now apply the character expansion method laid out in Sec. III. Let us expand the integrand in terms of supercharacters and use the orthogonality of representation matrix elements,

$$
\begin{aligned}
\mathcal{I}_{\mathrm{LS}} & =\int d \mu(U) \exp (\beta \operatorname{Str}(A U)) \exp \left(\beta \operatorname{Str}\left(B U^{-1}\right)\right) \\
& =\int d \mu(U)\left(\sum_{t} \frac{\sigma_{t}}{|t| !} \beta^{|t|} \xi_{t}(A U)\right)\left(\sum_{t^{\prime}} \frac{\sigma_{t^{\prime}}}{\left|t^{\prime}\right| !} \beta^{\left|t^{\prime}\right|} \xi_{t^{\prime}}\left(B U^{-1}\right)\right) \\
& =\sum_{t, t^{\prime}} \frac{\sigma_{t} \sigma_{t^{\prime}}}{|t| !\left|t^{\prime}\right| !} \beta^{|t|+\left|t^{\prime}\right|} \Gamma_{i j}^{t}(A) \Gamma_{l m}^{t^{\prime}}(B)(-1)^{\epsilon(i)+\epsilon(l)+(\epsilon(i)+\epsilon(j))(\epsilon(l)+\epsilon(m))} \underbrace{\int_{t}}_{=\delta_{t t^{\prime} \delta_{j l} \delta_{i m}(-1)^{\epsilon(m)} \alpha_{t}} \int d \mu(U) \Gamma_{j i}^{t}(U) \Gamma_{m l}^{t^{\prime}}\left(U^{-1}\right)} \\
& =\sum_{t}\left(\frac{\sigma_{t}}{|t| !} \beta^{|t|}\right)^{2} \alpha_{t} \xi_{t}(A B)
\end{aligned}
$$

where the sums are over all covariant representations $t, t^{\prime}$ and $\Gamma^{t}(U)$ is the representation matrix of $U$ corresponding to the Young diagram $t$. As already pointed out in Sec. III, we can restrict the sum over the covariant representations $t$ to the non-degenerate ones, for which $\alpha_{t} \neq 0$. The sum over all non-degenerate representations can be expressed by two sums over all sub-diagrams $p$ and $q$ corresponding to the ordinary groups $\mathrm{Gl}(m)$ and $\mathrm{Gl}(n)$, respectively. Inserting the norm given in Eq. (18) and the supercharacter given in Eq. (14) yields

$$
\mathcal{I}_{\mathrm{LS}}=\mathcal{C}_{m} \mathcal{C}_{n} \sum_{p, q} \frac{1}{(|p|+|q|+m n) !} \sigma_{\substack{\{m \times n\}\{p\} \\\left\{q^{T}\right\}}}\left(\prod_{i=1}^{m+n} \frac{1}{k_{i} !}\right) \Sigma\left(\lambda^{2} ; m, n\right) \chi_{p}\left(\lambda_{1}^{2}, \ldots, \lambda_{m}^{2}\right) \chi_{q}\left(\lambda_{m+1}^{2}, \ldots, \lambda_{m+n}^{2}\right)
$$

where $\lambda_{1}^{2}, \ldots, \lambda_{m+n}^{2}$ are the eigenvalues of the supermatrix $A B$ and

$$
\frac{\sigma_{p}}{|p| ! d_{p}} \frac{\sigma_{q}}{|q| ! d_{q}}=\mathcal{C}_{m} \mathcal{C}_{n}\left(\prod_{i=1}^{m+n} \frac{1}{k_{i} !}\right)
$$




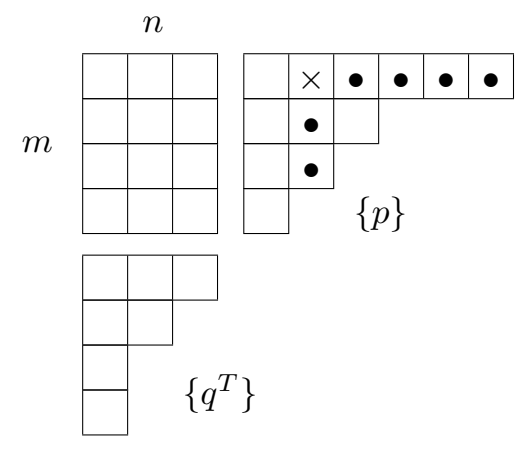

FIG. 2: Young diagram for a representation $t$ with a hook of length 7 .

Note that we have set $\beta=1$, as we can reinstate it later by a redefinition of $A$ and $B$. The term

$$
\frac{\sigma_{t}}{|t| !}=\frac{1}{(|p|+|q|+m n) !} \sigma_{\substack{\{m \times n\} \\\left\{q^{T}\right\}}}
$$

can be decomposed in terms of the sub-diagrams $p$ and $q$ using the hook length formula

$$
\frac{\sigma_{t}}{|t| !}=\prod_{i j} \frac{1}{h_{i j}}
$$

where the product is over all boxes in the diagram $t$. This formula can be found in standard textbooks. The hook length $h_{i j}$ of the $j$-th box in the $i$-th line of the diagram is defined as the number of boxes to the right of this box plus the number of boxes below this box plus one, cf. Fig. 2. The decomposed $\sigma_{t}$ reads

$$
\begin{aligned}
\frac{\sigma_{t}}{|t| !} & =\frac{\sigma_{p}}{|p| !} \frac{\sigma_{q}}{|q| !}\left(\prod_{i=1}^{m} \prod_{j=1}^{n} \frac{1}{m+n+1+p_{i}-i+q_{j}-j}\right) \\
& =\frac{\sigma_{p}}{|p| !} \frac{\sigma_{q}}{|q| !}\left(\prod_{i=1}^{m} \prod_{j=1}^{n} \frac{1}{k_{i}+k_{m+j}+1}\right) .
\end{aligned}
$$

Putting the pieces together we obtain

$$
\begin{aligned}
\mathcal{I}_{\mathrm{LS}}= & \mathcal{C}_{m} \mathcal{C}_{n} \sum_{p, q} \frac{\Delta\left(k_{1}, \ldots, k_{m}\right) \Delta\left(k_{m+1}, \ldots, k_{m+n}\right)}{\left(k_{1} !\right)^{2} \cdots\left(k_{m+n} !\right)^{2}}\left(\prod_{i=1}^{m} \prod_{j=1}^{n} \frac{1}{k_{i}+k_{m+j}+1}\right) \\
& \times \Sigma\left(\lambda^{2} ; m, n\right) \chi_{p}\left(\lambda_{1}^{2}, \ldots, \lambda_{m}^{2}\right) \chi_{q}\left(\lambda_{m+1}^{2}, \ldots, \lambda_{m+n}^{2}\right) .
\end{aligned}
$$

Using Weyl's character formula, given in Eq. [16, we write the sums over $p$ and $q$ as sums over $k_{1}, \ldots, k_{m+n}$. We then apply Theorem C.1 and are left with

$$
\begin{aligned}
\mathcal{I}_{\mathrm{LS}}= & \mathcal{C}_{m} \mathcal{C}_{n} \frac{\Sigma\left(\lambda^{2} ; m, n\right)}{\Delta\left(\lambda_{1}^{2}, \ldots, \lambda_{m}^{2}\right) \Delta\left(\lambda_{m+1}^{2}, \ldots, \lambda_{m+n}^{2}\right)} \\
& \times \sum_{k_{1}, \ldots, k_{m+n}=0}^{\infty} \frac{\Delta\left(k_{1}, \ldots, k_{m}\right) \Delta\left(k_{m+1}, \ldots, k_{m+n}\right)}{\left(k_{1} !\right)^{2} \cdots\left(k_{m+n} !\right)^{2}}\left(\prod_{i=1}^{m} \prod_{j=1}^{n} \frac{1}{k_{i}+k_{m+j}+1}\right) \lambda_{1}^{2 k_{1}} \cdots \lambda_{m+n}^{2 k_{m+n}} .
\end{aligned}
$$

We now use a power series identity which is conjectured in App. D It states that the two power series $\mathcal{J}_{0}$ and $\mathcal{J}_{m}$ of $N$ complex variables $z_{1}, \ldots, z_{N}$, defined by

$$
\mathcal{J}_{0} \equiv \sum_{k_{1}^{\prime}, \ldots, k_{N}^{\prime}=0}^{\infty} \frac{\Delta\left(k_{1}^{\prime}, \ldots, k_{N}^{\prime}\right)}{\left(k_{1}^{\prime} !\right)^{2} \cdots\left(k_{N}^{\prime} !\right)^{2}} z_{1}^{k_{1}^{\prime}} \cdots z_{N}^{k_{N}^{\prime}}
$$


and

$$
\mathcal{J}_{m} \equiv \sum_{k_{1}, \ldots, k_{N}=0}^{\infty} \frac{\Delta\left(k_{1}, \ldots, k_{m}\right) \Delta\left(k_{m+1}, \ldots, k_{N}\right)}{\left(k_{1} !\right)^{2} \cdots\left(k_{N} !\right)^{2}}\left(\prod_{i=1}^{m} \prod_{j=m+1}^{N} \frac{z_{i}-z_{j}}{k_{i}+k_{j}+1}\right) z_{1}^{k_{1}} \cdots z_{N}^{k_{N}},
$$

are identical for all $m$ with $1 \leq m \leq N$. Unfortunately, we do not have an analytical proof for this identity for arbitrary $N$. Explicit proofs are given for $N=2$ and $N=3$ in Apps. D and E In App. D we present compelling numerical and partial analytical evidence in favor of the conjecture for arbitrary $N$, which leaves very little doubt that the conjecture is correct. Appendix Dalso discusses a connection of the identity to Richardson-Littlewood coefficients.

Inserting the conjectured identity $\mathcal{J}_{0}=\mathcal{J}_{m}$ in Eq. $(29)$, we obtain

$$
\mathcal{I}_{\mathrm{LS}}=\mathcal{C}_{m} \mathcal{C}_{n} \frac{1}{\Delta\left(\lambda_{1}^{2}, \ldots, \lambda_{m}^{2}\right) \Delta\left(\lambda_{m+1}^{2}, \ldots, \lambda_{m+n}^{2}\right)} \sum_{k_{1}, \ldots, k_{m+n}=0}^{\infty} \frac{\Delta\left(k_{1}, \ldots, k_{m+n}\right)}{\left(k_{1} !\right)^{2} \cdots\left(k_{m+n} !\right)^{2}} \lambda_{1}^{2 k_{1}} \cdots \lambda_{m+n}^{2 k_{m+n}}
$$

Applying Theorems C.1 and C.3 then yields

$$
\begin{aligned}
\mathcal{I}_{\mathrm{LS}}= & \mathcal{C}_{m} \mathcal{C}_{n} \frac{1}{\Delta\left(\lambda_{1}^{2}, \ldots, \lambda_{m}^{2}\right) \Delta\left(\lambda_{m+1}^{2}, \ldots, \lambda_{m+n}^{2}\right)} \\
& \times \sum_{k_{1}>k_{2}>\cdots>k_{m+n} \geq 0} \operatorname{det}\left(\frac{1}{k_{j} !\left(k_{j}-m-n+i\right) !}\right)_{i, j=1, \ldots, m+n} \operatorname{det}\left(\lambda_{i}^{2 k_{j}}\right)_{i, j=1, \ldots, m+n} .
\end{aligned}
$$

Using Theorem C.2 and the power series expansion of the Bessel function [39,

$$
\lambda^{-\nu} I_{\nu}(2 \lambda)=\sum_{k=0}^{\infty} \frac{\lambda^{2 k}}{k !(k+\nu) !}
$$

we find

$$
\begin{aligned}
\mathcal{I}_{\mathrm{LS}} & =\mathcal{C}_{m} \mathcal{C}_{n} \frac{\operatorname{det}\left(\lambda_{j}^{m+n-i} I_{i-m-n}\left(2 \lambda_{j}\right)\right)_{i, j=1, \ldots, m+n}}{\Delta\left(\lambda_{1}^{2}, \ldots, \lambda_{m}^{2}\right) \Delta\left(\lambda_{m+1}^{2}, \ldots, \lambda_{m+n}^{2}\right)} \\
& =\mathcal{C}_{m} \mathcal{C}_{n} \frac{\operatorname{det}\left(\lambda_{j}^{m+n-i} I_{m+n-i}\left(2 \lambda_{j}\right)\right)_{i, j=1, \ldots, m+n}}{\Delta\left(\lambda_{1}^{2}, \ldots, \lambda_{m}^{2}\right) \Delta\left(\lambda_{m+1}^{2}, \ldots, \lambda_{m+n}^{2}\right)},
\end{aligned}
$$

where we used $I_{\nu}=I_{-\nu}$ in the last line. Finally, we reinstate $\beta$ by rescaling the $\lambda_{i}$ and obtain

$$
\mathcal{I}_{\mathrm{LS}}=\mathcal{C}_{m} \mathcal{C}_{n} \beta^{\frac{(m+n)-(m-n)^{2}}{2}} \frac{\operatorname{det}\left(\lambda_{j}^{m+n-i} I_{m+n-i}\left(2 \beta \lambda_{j}\right)\right)_{i, j=1, \ldots, m+n}}{\Delta\left(\lambda_{1}^{2}, \ldots, \lambda_{m}^{2}\right) \Delta\left(\lambda_{m+1}^{2}, \ldots, \lambda_{m+n}^{2}\right)} .
$$

To the best of our knowledge this result has not been derived before.

\section{B. Extended Supersymmetric Berezin-Karpelevich Integral}

We now calculate the extension of the Berezin-Karpelevich integral over $U, V \in \mathrm{U}(m \mid n)$ defined in Sec. II

$$
\mathcal{I}_{\mathrm{BK}} \equiv \int d \mu(U) \int d \mu(V) \exp \left(\beta \operatorname{Str}\left(U A V B+U^{-1} C V^{-1} D\right)\right)
$$

where again the notation is as in Sec. II] We expand the integrand in terms of supercharacters and obtain

$$
\mathcal{I}_{\mathrm{BK}}=\sum_{t, t^{\prime}} \frac{\sigma_{t} \sigma_{t^{\prime}}}{|t| !\left|t^{\prime}\right| !} \beta^{|t|+\left|t^{\prime}\right|} \int d \mu(U) \int d \mu(V) \xi_{t}(U A V B) \xi_{t^{\prime}}\left(U^{-1} C V^{-1} D\right)
$$

where the sum is over all covariant representations of $\operatorname{Gl}(m \mid n)$. We now insert the definition of the supercharacter as the supertrace of the corresponding representation matrix and make use of the orthogonality of representation matrix elements. After a careful handling of signs due to supermatrix element commutation, we obtain

$$
\mathcal{I}_{\mathrm{BK}}=\sum_{t}\left(\frac{\sigma_{t} \alpha_{t}}{|t| !} \beta^{|t|}\right)^{2} \xi_{t}(B C) \xi_{t}(A D) .
$$


We again replace the sum over all covariant representations $t$ by two sums over all representations $p$ and $q$ of the ordinary groups $\mathrm{Gl}(m)$ and $\mathrm{Gl}(n)$, respectively, as the terms we omit in doing so are terms with $\alpha_{t}=0$, which do not contribute to the result. Inserting Eqs. (18) and $(14)$, we obtain

$$
\begin{aligned}
\mathcal{I}_{\mathrm{BK}}= & \Sigma\left(\lambda^{2} ; m, n\right) \Sigma\left(\mu^{2} ; m, n\right) \sum_{p, q}\left(\frac{\beta^{|q|+|p|+m n}}{|q| !|p| !} \frac{\sigma_{p} \sigma_{q}}{d_{p} d_{q}}\right)^{2} \\
& \times \chi_{p}\left(\lambda_{1}^{2}, \ldots, \lambda_{m}^{2}\right) \chi_{p}\left(\mu_{1}^{2}, \ldots, \mu_{m}^{2}\right) \chi_{q}\left(\lambda_{m+1}^{2}, \ldots, \lambda_{m+n}^{2}\right) \chi_{q}\left(\mu_{m+1}^{2}, \ldots, \mu_{m+n}^{2}\right) \\
= & \Sigma\left(\lambda^{2} ; m, n\right) \Sigma\left(\mu^{2} ; m, n\right) \beta^{2 m n}\left[\sum_{p} \frac{\beta^{2|p|} \sigma_{p}^{2}}{d_{p}^{2}(|p| !)^{2}} \chi_{p}\left(\lambda_{1}^{2}, \ldots, \lambda_{m}^{2}\right) \chi_{p}\left(\mu_{1}^{2}, \ldots, \mu_{m}^{2}\right)\right] \\
& \times\left[\sum_{q} \frac{\beta^{2|q|} \sigma_{q}^{2}}{d_{q}^{2}(|q| !)^{2}} \chi_{q}\left(\lambda_{m+1}^{2}, \ldots, \lambda_{m+n}^{2}\right) \chi_{q}\left(\mu_{m+1}^{2}, \ldots, \mu_{m+n}^{2}\right)\right],
\end{aligned}
$$

where $\lambda_{1}^{2}, \ldots, \lambda_{m+n}^{2}$ are the eigenvalues of the matrix $B C$ and $\mu_{1}^{2}, \ldots, \mu_{m+n}^{2}$ are the eigenvalues of the matrix $A D$, respectively. According to Eq. (21) of Ref. [27, we find that the terms in brackets are nothing but ordinary BerezinKarpelevich integrals over groups $\mathrm{U}(m)$ and $\mathrm{U}(n)$, for which the result is given in Ref. [27]. We thus obtain

$$
\mathcal{I}_{\mathrm{BK}}=\mathcal{C}_{m}^{2} \mathcal{C}_{n}^{2} \beta^{(m+n)-(m-n)^{2}} \frac{\operatorname{det}\left(I_{0}\left(2 \beta \lambda_{i} \mu_{j}\right)\right)_{i, j=1, \ldots, m} \operatorname{det}\left(I_{0}\left(2 \beta \lambda_{m+i} \mu_{m+j}\right)\right)_{i, j=1, \ldots, n}}{B\left(\lambda^{2} ; m, n\right) B\left(\mu^{2} ; m, n\right)}
$$

with the Berezinian

$$
B\left(\lambda^{2} ; m, n\right)=\frac{\Delta\left(\lambda_{1}^{2}, \ldots, \lambda_{m}^{2}\right) \Delta\left(\lambda_{m+1}^{2}, \ldots, \lambda_{m+n}^{2}\right)}{\prod_{i=1}^{m} \prod_{j=1}^{n}\left(\lambda_{i}^{2}-\lambda_{m+j}^{2}\right)} .
$$

This extends the result obtained in Ref. [13] using the diffusion equation method by introducing two additional independent supermatrix parameters.

\section{SUMMARY AND OUTLOOK}

We have extended the character expansion method to supergroup integrals and calculated a supersymmetric Leutwyler-Smilga integral that, to the best of our knowledge, has not been known before. In the course of the calculation of this integral, we used a conjecture which, unfortunately, lacks a complete proof. However, in App. D we presented strong arguments that this conjecture is correct. We also calculated a supersymmetric Berezin-Karpelevich integral extending the result obtained in Ref. [13].

The character expansion method developed in this paper should allow for the calculation of other supergroup integrals that might not be calculable using other methods such as the diffusion equation method 13 . In the case of the ordinary Leutwyler-Smilga integral, a determinant term can be included in the integrand as well [14, 27, 40, The inclusion of a superdeterminant term is also of interest in the supersymmetric version of the integral. Work in this direction is in progress.

\section{Acknowledgments}

CL would like to thank Andreas Schäfer, who suggested the main idea of App. D 2. TG acknowledges support from Deutsche Forschungsgemeinschaft (Sonderforschungsbereich Transregio 12, "Symmetries and Universality in Mesoscopic Systems"). YW acknowledges support from EPSRC grant EP/C515056/1 ("Random Matrices and Polynomials: a tool to understand complexity").

\section{APPENDIX A: EXTENSION TO NON-DIAGONALIZABLE SUPERMATRICES}

The integral formulas presented in Sec. II can be extended to non-diagonalizable supermatrices by a limiting procedure. In this section we demonstrate the problem of non-diagonalizable supermatrices in the case of $1+1$ dimensions. The results can be generalized in a straightforward way to higher dimensions. 
Let $M$ be an arbitrary $(1+1)$-dimensional supermatrix,

$$
M=\left(\begin{array}{ll}
a & \alpha \\
\beta & b
\end{array}\right)
$$

where $a, b$ are even elements of the algebra and $\alpha, \beta$ are odd elements of the algebra. If $(a-b)^{-1}$ exists, i.e., $a$ and $b$ are not equal up to nilpotent terms, $M$ can be diagonalized,

$$
M \equiv V_{M}^{-1} M_{D} V_{M},
$$

where

$$
\begin{aligned}
V_{M} & =\left(\begin{array}{cc}
1-\frac{\alpha \beta}{2(a-b)^{2}} & -\frac{\alpha}{a-b} \\
\frac{\beta}{a-b} & 1+\frac{\alpha \beta}{2(a-b)^{2}}
\end{array}\right), \\
V_{M}^{-1} & =\left(\begin{array}{cc}
1-\frac{\alpha \beta}{2(a-b)^{2}} & \frac{\alpha}{a-b} \\
-\frac{\beta}{a-b} & 1+\frac{\alpha \beta}{2(a-b)^{2}}
\end{array}\right), \\
M_{D} & =\left(\begin{array}{cc}
a+\frac{\alpha \beta}{a-b} & 0 \\
0 & b+\frac{\alpha \beta}{a-b}
\end{array}\right) .
\end{aligned}
$$

The entries of $M_{D}$ are the (geometric) eigenvalues of $M$.

We would like to briefly comment on the algebraic definition of eigenvalues as solutions of the characteristic equations $\operatorname{Sdet}(M-m)=0$ and $\operatorname{Sdet}(M-m)^{-1}=0$. In the present case the algebraic definition has four solutions,

$$
\begin{aligned}
& m_{1}=a+\frac{\alpha \beta}{a-b}, \\
& m_{2}=b+\frac{\alpha \beta}{a-b}, \\
& m_{3}=b-\frac{\alpha \beta}{a-b}, \\
& m_{4}=a-\frac{\alpha \beta}{a-b} .
\end{aligned}
$$

Only two of these coincide with the geometric eigenvalues. Therefore a consistent definition of eigenvalues is only possible if a supermatrix is diagonalizable. We assume the geometric definition of eigenvalues in this paper. In general a supermatrix cannot be diagonalized if one of the eigenvalues of its boson-boson block coincides with one of the eigenvalues of its fermion-fermion block up to nilpotent terms. This problem looks severe at first sight, but although the limit $b \rightarrow a$ of the eigenvalues does not exist, the supersymmetric integrals have well-defined limits.

Let us consider the case of $b=a+\varepsilon$ with $\varepsilon \rightarrow 0$. The case of $b \rightarrow a+\hat{n}$, where $\hat{n}$ is nilpotent, can be obtained in the same way by keeping higher orders in $\varepsilon$ and substituting $\hat{n}$ for $\varepsilon$ in the end.

In the case of the supersymmetric Leutwyler-Smilga integral given in Eq. (2) with $A B=M$, we find

$$
\left.I_{0}\left(\lambda_{1}\right) I_{1}\left(\lambda_{2}\right) \lambda_{2}-I_{0}\left(\lambda_{2}\right) I_{1}\left(\lambda_{1}\right) \lambda_{1} \rightarrow-\frac{\alpha \beta}{2 a} I_{1}(\sqrt{a})\right)^{2} .
$$

The supersymmetric Berezin-Karpelevich integral given in Eq. (5) with $B C=M$ also has a well-defined limit for $b \rightarrow a$ with

$$
\left(\lambda_{1}^{2}-\lambda_{2}^{2}\right) I_{0}\left(\lambda_{1} \mu_{1}\right) I_{0}\left(\lambda_{2} \mu_{2}\right) \rightarrow \frac{\alpha \beta}{2 \sqrt{a}}\left(I_{0}\left(\sqrt{a} \mu_{2}\right) I_{1}\left(\sqrt{a} \mu_{1}\right) \mu_{1}+I_{0}\left(\sqrt{a} \mu_{1}\right) I_{1}\left(\sqrt{a} \mu_{2}\right) \mu_{2}\right) .
$$

As mentioned above, this limiting procedure can be generalized to higher dimensions.

\section{APPENDIX B: THE LIMIT OF COINCIDING EIGENVALUES}

The results given in Eqs. (2) and (5) require a limiting procedure if two eigenvalues within the first $m$ or within the last $n$ eigenvalues coincide. We calculate these limits explicitly in this section. 
We first consider the result of the supersymmetric Leutwyler-Smilga integral,

$$
\mathcal{I}_{\mathrm{LS}}=\mathcal{C}_{m} \mathcal{C}_{n} \beta^{\frac{(m+n)-(m-n)^{2}}{2}} \frac{\operatorname{det}\left(\lambda_{j}^{m+n-i} I_{m+n-i}\left(2 \beta \lambda_{j}\right)\right)_{i, j=1, \ldots, m+n}}{\Delta\left(\lambda_{1}^{2}, \ldots, \lambda_{m}^{2}\right) \Delta\left(\lambda_{m+1}^{2}, \ldots, \lambda_{m+n}^{2}\right)}
$$

If one of the bosonic eigenvalues $\left(\lambda_{1}, \ldots, \lambda_{m}\right)$ coincides with one of the fermionic eigenvalues $\left(\lambda_{m+1}, \ldots, \lambda_{m+n}\right)$, the integral vanishes. The case of two coinciding eigenvalues within bosonic or fermionic eigenvalues is slightly more involved. Let us assume, without loss of generality, that the two coinciding eigenvalues are $\lambda_{1}$ and $\lambda_{2}$. We analyze the limit $\lambda_{2}-\lambda_{1} \equiv \varepsilon \rightarrow 0$. Up to linear order in $\varepsilon$, we find

$$
\begin{aligned}
\lambda_{2}^{m+n-i} I_{m+n-i}\left(2 \beta \lambda_{2}\right) & =\left(\lambda_{1}+\varepsilon\right)^{m+n-i} I_{m+n-i}\left(2 \beta\left(\lambda_{1}+\varepsilon\right)\right) \\
& =\lambda_{1}^{m+n-i} I_{m+n-i}\left(2 \beta \lambda_{1}\right)+2 \beta \varepsilon \lambda_{1}^{m+n-i} I_{m+n-i-1}\left(2 \beta \lambda_{1}\right)
\end{aligned}
$$

and

$$
\Delta\left(\lambda_{1}^{2}, \ldots, \lambda_{m}^{2}\right)=-2 \varepsilon \lambda_{1} \Delta\left(\lambda_{2}^{2}, \ldots, \lambda_{m}^{2}\right) \prod_{i=3}^{m}\left(\lambda_{1}^{2}-\lambda_{i}^{2}\right) .
$$

Therefore the result for $\lambda_{2}=\lambda_{1}$ is obtained by replacing the second column of the matrix in the numerator by

$$
\beta \lambda_{1}^{m+n-i-1} I_{m+n-i-1}\left(2 \beta \lambda_{1}\right)
$$

and the first Vandermonde determinant in the denominator by

$$
-\Delta\left(\lambda_{2}^{2}, \ldots, \lambda_{m}^{2}\right) \prod_{i=3}^{m}\left(\lambda_{1}^{2}-\lambda_{i}^{2}\right) .
$$

The supersymmetric Berezin-Karpelevich integral

$$
\mathcal{I}_{\mathrm{BK}}=\mathcal{C}_{m}^{2} \mathcal{C}_{n}^{2} \beta^{(m+n)-(m-n)^{2}} \frac{\operatorname{det}\left(I_{0}\left(2 \beta \lambda_{i} \mu_{j}\right)\right)_{i, j=1, \ldots, m} \operatorname{det}\left(I_{0}\left(2 \beta \lambda_{m+i} \mu_{m+j}\right)\right)_{i, j=1, \ldots, n}}{B\left(\lambda^{2} ; m, n\right) B\left(\mu^{2} ; m, n\right)}
$$

also vanishes if one of the bosonic eigenvalues $\left(\lambda_{1}, \ldots, \lambda_{m}\right.$ resp. $\left.\mu_{1}, \ldots, \mu_{m}\right)$ coincides with one of the corresponding fermionic eigenvalues $\left(\lambda_{m+1}, \ldots, \lambda_{m+n}\right.$ resp. $\left.\mu_{m+1}, \ldots, \mu_{m+n}\right)$. For the case of two coinciding eigenvalues within bosonic or fermionic eigenvalues, we again consider $\lambda_{2}=\lambda_{1}+\varepsilon$ with $\varepsilon \rightarrow 0$. Up to linear order in $\varepsilon$,

$$
I_{0}\left(2 \beta \lambda_{2} \mu_{j}\right)=I_{0}\left(2 \beta \lambda_{1} \mu_{j}\right)+2 \beta \mu_{j} \varepsilon I_{1}\left(2 \beta \lambda_{1} \mu_{j}\right) .
$$

For $\lambda_{2}=\lambda_{1}$ the result is therefore obtained by replacing the second row of the first matrix in the numerator by

$$
\beta \mu_{j} \lambda_{1}^{-1} I_{-1}\left(2 \beta \lambda_{1} \mu_{j}\right)
$$

and the first Vandermonde determinant in the first Berezinian in the denominator by Eq. (B4).

This procedure can be repeated in the case of multiple coinciding eigenvalues in a straightforward way.

\section{APPENDIX C: ALGEBRAIC THEOREMS}

In the following theorems, $p_{1}, \ldots, p_{N}$ is a permutation of $1, \ldots, N$ and $\sigma_{\{p\}}$ is the sign of the permutation (positive if an even number of neighbors were exchanged, negative otherwise).

Theorem C.1. If $A_{k_{1}, \ldots, k_{N}}=\sigma_{\{p\}} A_{k_{p_{1}}, \ldots, k_{p_{N}}}$, then

$$
\sum_{k_{1}, \ldots, k_{N}=0}^{\infty} A_{k_{1}, \ldots, k_{N}} a_{1}^{k_{1}} \cdots a_{N}^{k_{N}}=\sum_{k_{1}>k_{2}>\cdots>k_{N} \geq 0} A_{k_{1}, \ldots, k_{N}} \operatorname{det}\left(a_{i}^{k_{j}}\right)_{i, j=1, \ldots, N} .
$$


Proof.

$$
\begin{aligned}
\sum_{k_{1}, \ldots, k_{N}=0}^{\infty} A_{k_{1}, \ldots, k_{N}} a_{1}^{k_{1}} \cdots a_{N}^{k_{N}} & =\frac{1}{N !} \sum_{\{p\}} \sum_{k_{1}, \ldots, k_{N}=0}^{\infty} \underbrace{A_{k_{p_{1}}, \ldots, k_{p_{N}}}}_{=\sigma_{\{p\}} A_{k_{1}, \ldots, k_{N}}} a_{1}^{k_{p_{1}}} \cdots a_{N}^{k_{p_{N}}} \\
& =\frac{1}{N !} \sum_{k_{1}, \ldots, k_{N}=0}^{\infty} A_{k_{1}, \ldots, k_{N}} \underbrace{\sum_{\{p\}} \sigma_{\{p\}} a_{1}^{k_{p_{1}}} \cdots a_{N}^{k_{p_{N}}}}_{=\operatorname{det}\left(a_{i}^{k_{j}}\right)_{i, j=1, \ldots, N}}
\end{aligned}
$$

Now the summand is invariant under permutation of the $k_{i}$, so instead of averaging over all permutations, we can pick one specific order. Noting that the summand vanishes for a pair of equal $k$, the proof is complete.

The following theorem was taken from Ref. [8].

Theorem C.2. Let the power series

$$
f_{i}(z)=\sum_{k=0}^{\infty} a_{k}^{i} z^{k}
$$

converge for $|z|<\rho$. Then,

$$
\operatorname{det}\left(f_{i}\left(z_{j}\right)\right)_{i, j=1, \ldots, N}=\sum_{k_{1}>k_{2}>\cdots>k_{N} \geq 0} \operatorname{det}\left(a_{k_{j}}^{i}\right)_{i, j=1, \ldots, N} \operatorname{det}\left(z_{i}^{k_{j}}\right)_{i, j=1, \ldots, N}
$$

for $\left|z_{i}\right|<\rho, i=1, \ldots, N$.

Proof.

$$
\begin{aligned}
\operatorname{det}\left(f_{i}\left(z_{j}\right)\right)_{i, j=1, \ldots, N} & =\sum_{\{p\}} \sigma_{\{p\}} f_{p_{1}}\left(z_{1}\right) \cdots f_{p_{N}}\left(z_{N}\right) \\
& =\sum_{\{p\}} \sigma_{\{p\}} \sum_{k_{1}, \ldots, k_{N}=0}^{\infty} a_{k_{1}}^{p_{1}} \cdots a_{k_{N}}^{p_{N}} z_{1}^{k_{1}} \cdots z_{N}^{k_{N}} \\
& =\sum_{k_{1}, \ldots, k_{N}=0} \underbrace{\left(\sum_{\{p\}} \sigma_{\{p\}} a_{k_{1}}^{p_{1}} \cdots a_{k_{N}}^{p_{N}}\right)}_{=\operatorname{det}\left(a_{k_{j}}^{i}\right)_{i, j=1, \ldots, N}} z_{1}^{k_{1}} \cdots z_{N}^{k_{N}} .
\end{aligned}
$$

We can now apply Theorem C.1, and the proof is complete.

Theorem C.3. Let the integers $k_{1}, \ldots, k_{N} \geq 0$ and $n_{1}, \ldots, n_{N} \geq 0$ be related through

$$
k_{j} \equiv n_{j}+N-j .
$$

Then,

$$
\operatorname{det}\left(\frac{1}{\left(n_{j}+i-j\right) !}\right)_{i, j=1, \ldots, N}=\frac{\Delta\left(k_{1}, \ldots, k_{N}\right)}{\prod_{i=1}^{N} k_{i} !}
$$

where

$$
\Delta\left(k_{1}, \ldots, k_{N}\right) \equiv \prod_{1 \leq i<j \leq N}\left(k_{i}-k_{j}\right)=\operatorname{det}\left(k_{i}^{N-j}\right)_{i, j=1, \ldots, N}
$$

Note that terms with factorials of negative integers in the denominator have to be understood as equal to zero. 
Proof. By rearranging the columns of the matrices in question it is straightforward to see that the statement is equivalent to

$$
\operatorname{det}\left(\frac{k_{j} !}{\left(k_{j}-i+1\right) !}\right)_{i, j=1, \ldots, N}=\operatorname{det}\left(k_{i}^{j-1}\right)_{i, j=1, \ldots, N} .
$$

Let us define two matrices $A$ and $C$ with elements

$$
\begin{aligned}
A_{i j} & \equiv k_{j}^{i-1} \\
C_{i j} & \equiv \frac{k_{j} !}{\left(k_{j}-i+1\right) !} .
\end{aligned}
$$

Because of the structure of $C_{i j}$ it is always possible to find a triangular matrix $B$ with diagonal entries $1, \ldots, 1$ that satisfies

$$
C=B A
$$

We thus obtain

$$
\operatorname{det}(C)=\underbrace{\operatorname{det}(B)}_{=1} \operatorname{det}(A)=\operatorname{det}(A)
$$

which completes the proof.

\section{APPENDIX D: CONJECTURE OF A POWER SERIES IDENTITY}

\section{Statement of the conjecture and discussion}

Conjecture D.1. Let two power series $\mathcal{J}_{0}$ and $\mathcal{J}_{m}$ of $N$ complex variables $z_{1}, \ldots, z_{N}$ be defined by

$$
\mathcal{J}_{0} \equiv \sum_{k_{1}^{\prime}, \ldots, k_{N}^{\prime}=0}^{\infty} \frac{\Delta\left(k_{1}^{\prime}, \ldots, k_{N}^{\prime}\right)}{\left(k_{1}^{\prime} !\right)^{2} \cdots\left(k_{N}^{\prime} !\right)^{2}} z_{1}^{k_{1}^{\prime}} \cdots z_{N}^{k_{N}^{\prime}}
$$

and

$$
\mathcal{J}_{m} \equiv \sum_{k_{1}, \ldots, k_{N}=0}^{\infty} \frac{\Delta\left(k_{1}, \ldots, k_{m}\right) \Delta\left(k_{m+1}, \ldots, k_{N}\right)}{\left(k_{1} !\right)^{2} \cdots\left(k_{N} !\right)^{2}}\left(\prod_{i=1}^{m} \prod_{j=m+1}^{N} \frac{z_{i}-z_{j}}{k_{i}+k_{j}+1}\right) z_{1}^{k_{1}} \cdots z_{N}^{k_{N}}
$$

where

$$
\Delta\left(a_{1}, \ldots, a_{N}\right) \equiv \prod_{1 \leq i<j \leq N}\left(a_{i}-a_{j}\right)
$$

is the Vandermonde determinant. Then,

$$
\mathcal{J}_{0}=\mathcal{J}_{m} \quad \text { for all } \quad 1 \leq m \leq N
$$

In the case of $N=2$ and $m=1$, the proof is straightforward,

$$
\begin{aligned}
\sum_{k_{1}, k_{2}=0}^{\infty} \frac{k_{1}-k_{2}}{\left(k_{1} !\right)^{2}\left(k_{2} !\right)^{2}} z_{1}^{k_{1}} z_{2}^{k_{2}} & =\sum_{k_{1}, k_{2}=0}^{\infty} \frac{k_{1}^{2}-k_{2}^{2}}{\left(k_{1} !\right)^{2}\left(k_{2} !\right)^{2}} \frac{1}{k_{1}+k_{2}} z_{1}^{k_{1}} z_{2}^{k_{2}} \\
& =\sum_{k_{1}, k_{2}=0}^{\infty} \frac{z_{1}-z_{2}}{\left(k_{1} !\right)^{2}\left(k_{2} !\right)^{2}} \frac{1}{k_{1}+k_{2}+1} z_{1}^{k_{1}} z_{2}^{k_{2}}
\end{aligned}
$$

Unfortunately, we have been unable to find an analytical proof for arbitrary $N$ and $m$. Partial analytical arguments are presented in App. D2 and a proof for $N=3$ is given in App. E, but the most compelling evidence in favor of the conjecture is due to numerical checks. Using complex random numbers for the $z_{i}$, we have checked the conjecture up to $N=25$ for all values of $m$ with $1 \leq m \leq N$. We employed the GNU multi-precision package 41] and found the conjecture to be satisfied to a precision of 2048 bits. In the absence of a complete analytical proof, this leads us to believe very strongly that the conjecture is indeed correct.

A connection of Conjecture D.1 to Richardson-Littlewood coefficients is discussed in App. D 3. 


\section{Proof for a subset of coefficients in the case of arbitrary $N$ and $m$}

In this section we give a proof of the conjecture for a subset of coefficients in the power series. We note that $\mathcal{J}_{0}$ is antisymmetric under the exchange of two of the $z_{i}$. Therefore, it is divisible by the Vandermonde determinant $\Delta\left(z_{1}, \ldots, z_{N}\right)$, which can be written as

$$
\Delta\left(z_{1}, \ldots, z_{N}\right)=\Delta\left(z_{1}, \ldots, z_{m}\right) \Delta\left(z_{m+1}, \ldots, z_{N}\right) \prod_{i=1}^{m} \prod_{j=m+1}^{N}\left(z_{i}-z_{j}\right) .
$$

Hence $\mathcal{J}_{0}$ is also divisible by $\prod_{i=1}^{m} \prod_{j=m+1}^{N}\left(z_{i}-z_{j}\right)$. The ansatz

$$
\mathcal{J}_{0}=\left(\prod_{i=1}^{m} \prod_{j=m+1}^{N}\left(z_{i}-z_{j}\right)\right) \sum_{k_{1}, \ldots, k_{N}=0}^{\infty} c_{k_{1}, \ldots, k_{N}} z_{1}^{k_{1}} \cdots z_{N}^{k_{N}} \equiv \tilde{\mathcal{J}}_{m}
$$

yields

$$
c_{k_{1}, \ldots, k_{N}} \equiv \Delta\left(k_{1}, \ldots, k_{m}\right) \Delta\left(k_{m+1}, \ldots, k_{N}\right) s_{k_{1}, \ldots, k_{N}}
$$

with $s_{k_{1}, \ldots, k_{N}}$ symmetric under the exchange of two of the $k_{i}$ in the subsets $\left\{k_{1}, \ldots, k_{m}\right\}$ or $\left\{k_{m+1}, \ldots, k_{N}\right\}$ in order to satisfy the symmetry requirements of $\mathcal{J}_{0}$ under exchange of the $z_{i}$.

In the following, we determine $s_{k_{1}, \ldots, k_{N}}$ in the special limit

$$
0<z_{1} \ll z_{2} \ll \ldots \ll z_{m-1} \ll z_{m+1} \ll z_{m+2} \ll \ldots \ll z_{N-1} \ll 1
$$

and $z_{m}$ and $z_{N}$ of the order of 1 . In this limit the exchange term simplifies to

$$
\prod_{i=1}^{m} \prod_{j=m+1}^{N}\left(z_{i}-z_{j}\right) \rightarrow(-1)^{(m-1)(N-m)}\left(z_{m}-z_{N}\right) z_{m}^{k_{N}^{0}} \prod_{j=m+1}^{N} z_{j}^{k_{m}^{0}}
$$

with

$$
\begin{aligned}
& k_{N}^{0} \equiv N-m-1, \\
& k_{m}^{0} \equiv m-1 .
\end{aligned}
$$

The dominating terms in the power series in Eqs. 31) and (D5) correspond to

$$
\begin{array}{ll}
k_{i}=i-1 & \text { for } i<m, \\
k_{j}=j-m-1 & \text { for } m<j<N, \\
k_{i}^{\prime}=i-1 & \text { for } i<m, \\
k_{j}^{\prime}=j-2 & \text { for } m<j<N
\end{array}
$$

with arbitrary $k_{m}, k_{N}, k_{m}^{\prime}, k_{N}^{\prime}$. We therefore find

$$
\begin{aligned}
\tilde{\mathcal{J}}_{m}= & (-1)^{(m-1)(N-m)}\left(z_{m}-z_{N}\right) \sum_{k_{m}=k_{m}^{0}, k_{N}=k_{N}^{0}}^{\infty} \Delta\left(k_{1}, \ldots, k_{m}\right) \Delta\left(k_{m+1}, \ldots, k_{N}\right) s_{k_{1}, \ldots, k_{N}} \\
& \times\left(\prod_{i=1}^{k_{m}^{0}} z_{i}^{k_{i}}\right)\left(\prod_{j=m+1}^{N-1} z_{j}^{k_{m}^{0}+k_{j}}\right) z_{m}^{k_{m}+k_{N}^{0}} z_{N}^{k_{N}+k_{m}^{0}} \\
\mathcal{J}_{0}= & \sum_{k_{m}^{\prime}, k_{N}^{\prime}=0}^{\infty} \frac{\Delta\left(k_{1}^{\prime}, \ldots, k_{N}^{\prime}\right)}{\left(k_{1}^{\prime} !\right)^{2} \cdots\left(k_{N}^{\prime} !\right)^{2}} z_{m}^{k_{m}^{\prime}} z_{N}^{k_{N}^{\prime}}\left(\prod_{i=1}^{k_{m}^{0}} z_{i}^{k_{i}}\right)\left(\prod_{j=m+1}^{N-1} z_{j}^{k_{m}^{0}+k_{j}}\right) .
\end{aligned}
$$

After inserting the fixed values of $k_{i}, k_{i}^{\prime}, k_{j}$, and $k_{j}^{\prime}$ for $i<m$ and $m<j<N$, we obtain that $\mathcal{J}_{0}=\tilde{\mathcal{J}}_{m}$ is equivalent to

$$
\begin{aligned}
\sum_{k_{m}^{\prime}, k_{N}^{\prime}=0}^{\infty} & \frac{\left(k_{N}^{\prime}-k_{m}^{\prime}\right)}{\Sigma_{0} k_{m}^{\prime} !\left(k_{m}^{\prime}-\left(k_{m}^{0}+k_{N}^{0}\right)\right) ! k_{N}^{\prime} !\left(k_{N}^{\prime}-\left(k_{m}^{0}+k_{N}^{0}\right)\right) !} z_{m}^{k_{m}^{\prime}} z_{N}^{k_{N}^{\prime}} \\
& =\left(z_{N}-z_{m}\right) \sum_{k_{m}=k_{m}^{0}, k_{N}=k_{N}^{0}}^{\infty} \frac{k_{m} ! k_{N} ! s_{0,1, \ldots, k_{m}^{0}-1, k_{m}, 0,1, \ldots, k_{N}^{0}-1, k_{N}}}{\left(k_{m}-k_{m}^{0}\right) !\left(k_{N}-k_{N}^{0}\right) !} z_{m}^{k_{m}+k_{N}^{0}} z_{N}^{k_{N}+k_{m}^{0}}
\end{aligned}
$$


with

$$
\Sigma_{0} \equiv 1 ! \cdots\left(k_{m}^{0}+k_{N}^{0}-1\right) ! 1 ! \cdots\left(k_{m}^{0}-1\right) ! 1 ! \cdots\left(k_{N}^{0}-1\right) !,
$$

where we used the fact that

$$
\Delta(1+c, 2+c, \ldots, N+c)=\left(\prod_{i=1}^{N-1} i !\right)(-1)^{\frac{N(N-1)}{2}}
$$

with an arbitrary constant $c$. Note that

$$
\frac{k_{N}-k_{m}}{k_{m} !\left(k_{m}-a\right) ! k_{N} !\left(k_{N}-a\right) !}=\frac{1}{\left(k_{N}+k_{m}-a\right)} \frac{k_{N}\left(k_{N}-a\right)-k_{m}\left(k_{m}-a\right)}{k_{m} !\left(k_{m}-a\right) ! k_{N} !\left(k_{N}-a\right) !} .
$$

Hence the left-hand side of Eq. (D13) can be written as

$$
\begin{aligned}
\sum_{k_{m}^{\prime}, k_{N}^{\prime}=0}^{\infty} & \frac{z_{m}^{k_{m}^{\prime}} z_{N}^{k_{N}^{\prime}}}{\left(k_{N}^{\prime}+k_{m}^{\prime}-\left(k_{m}^{0}+k_{N}^{0}\right)\right)} \frac{k_{N}^{\prime}\left(k_{N}^{\prime}-\left(k_{m}^{0}+k_{N}^{0}\right)\right)-k_{m}^{\prime}\left(k_{m}^{\prime}-\left(k_{m}^{0}+k_{N}^{0}\right)\right)}{k_{m}^{\prime} !\left(k_{m}^{\prime}-\left(k_{m}^{0}+k_{N}^{0}\right)\right) ! k_{N}^{\prime} !\left(k_{N}^{\prime}-\left(k_{m}^{0}+k_{N}^{0}\right)\right) !} \\
& =\sum_{k_{m}^{\prime}, k_{N}^{\prime}=0}^{\infty} \frac{\left(z_{N}-z_{k}\right)}{\left(k_{N}^{\prime}+k_{m}^{\prime}-\left(k_{m}^{0}+k_{N}^{0}\right)+1\right)} \frac{z_{m}^{k_{m}^{\prime}} z_{N}^{k_{N}^{\prime}}}{k_{m}^{\prime} !\left(k_{m}^{\prime}-\left(k_{m}^{0}+k_{N}^{0}\right)\right) ! k_{N}^{\prime} !\left(k_{N}^{\prime}-\left(k_{m}^{0}+k_{N}^{0}\right)\right) !}
\end{aligned}
$$

Comparing coefficients of powers of $z_{m}$ and $z_{N}$ in Eq. D13 now yields

$$
s_{0,1, \ldots, k_{m}^{0}-1, k_{m}, 0,1, \ldots, k_{N}^{0}-1, k_{N}}=\frac{1}{\left(k_{m} !\right)^{2}\left(k_{N} !\right)^{2}\left(k_{m}+k_{N}+1\right)} \frac{1}{\Sigma_{0}\left(\prod_{i=1}^{k_{N}^{0}}\left(k_{m}+i\right)\right)\left(\prod_{j=1}^{k_{m}^{0}}\left(k_{N}+j\right)\right)} .
$$

For the specific choice of $k_{1}, \ldots, k_{N}$ stated in Eq. D10 the desired general result,

$$
s_{k_{1}, \ldots, k_{N}}=\frac{1}{\left(k_{1} !\right)^{2} \cdots\left(k_{N} !\right)^{2}} \prod_{i=1}^{m} \prod_{j=m+1}^{N} \frac{1}{k_{i}+k_{j}+1},
$$

is equivalent to Eq. D13). This means that for an infinite subset of coefficients, defined by Eq. (D10), the identity holds.

\section{Conjecture D.1 and Richardson-Littlewood coefficients}

In this section we discuss a relation for Richardson-Littlewood coefficients that can be obtained from Conjecture D.1. Because of the antisymmetry of $\mathcal{J}_{0}$ and $\mathcal{J}_{m}$ under the exchange of two of the $z_{i}$, we conclude that both sides are divisible by $\Delta\left(z_{1}, \ldots, z_{N}\right)$. Therefore we define

$$
\begin{aligned}
\mathcal{J}_{0}^{\prime} \equiv \frac{\mathcal{J}_{0}}{\Delta\left(z_{1}, \ldots, z_{N}\right)}=\sum_{k_{1}>k_{2}>\cdots>k_{N} \geq 0} f_{k} \frac{\operatorname{det}\left(z_{i}^{k_{j}}\right)_{i, j=1, \ldots, N}}{\Delta\left(z_{1}, \ldots, z_{N}\right)} \\
\mathcal{J}_{m}^{\prime} \equiv \frac{\mathcal{J}_{m}}{\Delta\left(z_{1}, \ldots, z_{N}\right)}=\sum_{\substack{k_{1}^{a}>k_{2}^{a}>\cdots>k_{m}^{a} \geq 0 \\
k_{m+1}^{b}>k_{m+2}^{b}>\cdots>k_{N}^{b} \geq 0}} g_{k^{a} k^{b}} \frac{\operatorname{det}\left(z_{i}^{k_{j}^{a}}\right)_{i, j=1, \ldots, m}}{\Delta\left(z_{1}, \ldots, z_{m}\right)} \frac{\operatorname{det}\left(z_{m+i}^{k_{j}^{b}}\right)_{i, j=1, \ldots, n}}{\Delta\left(z_{m+1}, \ldots, z_{N}\right)},
\end{aligned}
$$

where we used Theorem C.1 $n \equiv N-m$ and defined

$$
\begin{aligned}
f_{k} & \equiv \frac{\Delta\left(k_{1}, \ldots, k_{N}\right)}{k_{1} !^{2} \cdots k_{N} !^{2}} \\
g_{k^{a} k^{b}} & \equiv \frac{\Delta\left(k_{1}^{a}, \ldots, k_{m}^{a}\right) \Delta\left(k_{1}^{b}, \ldots, k_{n}^{b}\right)}{k_{1}^{a} !^{2} \cdots k_{m}^{a} !^{2} k_{1}^{b} !^{2} \cdots k_{n}^{b} !^{2}}\left(\prod_{i=1}^{m} \prod_{j=1}^{n} \frac{1}{k_{i}^{a}+k_{j}^{b}+1}\right)
\end{aligned}
$$


With $k_{i}^{a} \equiv p_{i}+m-i, k_{i}^{b} \equiv q_{i}+n-i$, and $k_{i} \equiv r_{i}+N-i$ we can relate the $k_{i}$ to Young diagrams $p, q$, and $r$. Note that

$$
\frac{\operatorname{det}\left(z_{i}^{k_{j}}\right)_{i, j=1, \ldots, N}}{\Delta\left(z_{1}, \ldots, z_{N}\right)} \equiv S_{r}\left(z_{1}, \ldots, z_{N}\right)
$$

is the Schur function corresponding to partition $r$ [42. Hence $\mathcal{J}_{0}=\mathcal{J}_{m}$ resp. $\mathcal{J}_{0}^{\prime}=\mathcal{J}_{m}^{\prime}$ can be written as

$$
\sum_{r} f_{r} S_{r}\left(z_{1}, \ldots, z_{N}\right)=\sum_{p, q} g_{p q} S_{p}\left(z_{1}, \ldots, z_{m}\right) S_{q}\left(z_{m+1}, \ldots, z_{N}\right) .
$$

We now make use of the fact that Schur functions form a basis of the ring of symmetric functions, i.e., the Schur functions on the left-hand side can be expanded in Schur functions occurring on the right-hand side. The expansion coefficients are called Richardson-Littlewood coefficients $c_{\mu \nu}^{r}$ [42],

$$
S_{r}\left(z_{1}, \ldots, z_{N}\right)=\sum_{\mu \subseteq r, \nu}^{\prime} c_{\mu \nu}^{r} S_{\mu}\left(z_{1}, \ldots, z_{m}\right) S_{\nu}\left(z_{m+1}, \ldots, z_{N}\right)
$$

where the sum is over all Young diagrams $\mu$ and $\nu$ with

$$
\begin{aligned}
|\mu|+|\nu| & =|r|, \\
\mu_{i} & \leq r_{i} \text { for all } i .
\end{aligned}
$$

As Schur functions are linearly independent, we can compare coefficients in Eq. (D25) and find

$$
\sum_{r \supseteq p}^{\prime} f_{r} c_{p q}^{r}=g_{p q}
$$

where the sum is over all Young diagrams $r$ with $r_{i} \geq p_{i}$ for all $i$ and $|r|=|p|+|q|$. Thus Conjecture D.1 implies the relation given in Eq. D28) for Richardson-Littlewood coefficients.

\section{APPENDIX E: THE SUPERSYMMETRIC LEUTWYLER-SMILGA INTEGRAL FOR U(1|1) AND U(2|1)}

In this section we calculate the supersymmetric Leutwyler-Smilga integral, defined in Eq. (1), over U(1|1) and $\mathrm{U}(2 \mid 1)$ by explicit parametrization of the supergroup. This calculation is not only a non-trivial check of our general result, but also serves as a proof of the power series identity which is conjectured in App. D for $N=2$ and $N=3$.

The Leutwyler-Smilga integral with $\beta=1 / 2$ reads

$$
\mathcal{I} \equiv \int d \mu(U) e^{\frac{1}{2} \operatorname{Str}\left(A U+B U^{\dagger}\right)}
$$

where the integral is over $\mathrm{U}(m \mid n)$. To prove Conjecture D.1 it suffices to take $A$ and $B$ to be arbitrary diagonal supermatrices with entries $a_{1}, \ldots, a_{m+n}$ and $b_{1}, \ldots, b_{m+n}$.

We adopt the following convention for complex conjugation of anticommuting numbers,

$$
\begin{aligned}
\left(\chi_{1} \chi_{2}\right)^{*} & =\chi_{2}^{*} \chi_{1}^{*}, \\
\left(\chi_{1}^{*}\right)^{*} & =\chi_{1},
\end{aligned}
$$

and parametrize an element $U$ of the unitary supergroup $\mathrm{U}(m \mid n)$ as

$$
U=U_{o} U_{g}=\left(\begin{array}{cc}
U_{m} & 0 \\
0 & U_{n}
\end{array}\right) \exp \left(\begin{array}{cccccc}
0 & \cdots & 0 & i \alpha_{11} & \cdots & i \alpha_{1 n} \\
\vdots & & \vdots & \vdots & & \vdots \\
0 & \cdots & 0 & i \alpha_{m 1} & \cdots & i \alpha_{m n} \\
i \alpha_{11}^{*} & \cdots & i \alpha_{m 1}^{*} & 0 & \cdots & 0 \\
\vdots & & \vdots & \vdots & & \vdots \\
i \alpha_{1 n}^{*} & \cdots & i \alpha_{m n}^{*} & 0 & \cdots & 0
\end{array}\right)
$$


where $U_{m}$ and $U_{n}$ are ordinary unitary $m \times m$ and $n \times n$ matrices and $\alpha_{11}, \ldots, \alpha_{m n}$ are anticommuting variables. This generalizes the parametrization introduced in Refs. 43, 44] for $\mathrm{U}(1 \mid 1)$. It is also very similar to the parametrization of the super-Riemannian manifold $\mathrm{Gl}(m \mid 1)$ used in Ref. [45].

The invariant integration measure corresponding to this parametrization for the cases of $m=n=1$ and $m=2$, $n=1$ is of the form

$$
d \mu(U)=d \mu\left(U_{m}\right) d \mu\left(U_{m}\right) d \alpha_{11} d \alpha_{11}^{*} \ldots d \alpha_{m n} d \alpha_{m n}^{*} \mathcal{T}_{m, n}\left(\alpha_{11}, \ldots, \alpha_{m n}, \alpha_{11}^{*}, \ldots, \alpha_{m n}^{*}\right),
$$

where $d \mu\left(U_{m}\right)$ and $d \mu\left(U_{n}\right)$ are the invariant measures of the ordinary groups $\mathrm{U}(m)$ and $\mathrm{U}(n)$, and $\mathcal{T}_{m, n}$ is a function only of anticommuting variables. We find

$$
\begin{aligned}
\mathcal{T}_{1,1}\left(\alpha_{11}, \alpha_{11}^{*}\right) & =1 \\
\mathcal{T}_{2,1}\left(\alpha_{11}, \alpha_{21}, \alpha_{11}^{*}, \alpha_{21}^{*}\right) & =1-\frac{1}{3}\left(\alpha_{11} \alpha_{11}^{*}+\alpha_{21} \alpha_{21}^{*}\right) .
\end{aligned}
$$

The supertrace can be written as

$$
\operatorname{Str}\left(A U+B U^{\dagger}\right)=\operatorname{Str}\left(U_{g} A U_{o}+B U_{g}^{\dagger} U_{o}^{\dagger}\right)=\operatorname{Tr}\left(\tilde{A}_{m} U_{m}+\tilde{B}_{m} U_{m}^{\dagger}\right)-\operatorname{Tr}\left(\tilde{A}_{n} U_{n}+\tilde{B}_{n} U_{n}^{\dagger}\right)
$$

where $\tilde{A}_{m}$ and $\tilde{B}_{m}$ are the boson-boson blocks of the supermatrices $U_{g} A$ and $B U_{g}^{\dagger}$, and $\tilde{A}_{n}$ and $\tilde{B}_{n}$ are the fermionfermion blocks of the supermatrices $U_{g} A$ and $B U_{g}^{\dagger}$. The integral thus factorizes in the following way,

$$
\begin{aligned}
\mathcal{I}= & \int d \alpha_{11} d \alpha_{11}^{*} \cdots d \alpha_{m n} d \alpha_{m n}^{*} \mathcal{T}_{m, n}\left(\alpha_{11}, \ldots, \alpha_{m n}, \alpha_{11}^{*}, \ldots, \alpha_{m n}^{*}\right) \\
& \times\left(\int d \mu\left(U_{m}\right) e^{\frac{1}{2} \operatorname{Tr}\left(\tilde{A}_{m} U_{m}+\tilde{B}_{m} U_{m}^{\dagger}\right)}\right)\left(\int d \mu\left(U_{m}\right) e^{-\frac{1}{2} \operatorname{Tr}\left(\tilde{A}_{n} U_{n}+\tilde{B}_{n} U_{n}^{\dagger}\right)}\right) .
\end{aligned}
$$

We perform the ordinary Leutwyler-Smilga integrals over the groups $\mathrm{U}(m)$ and $\mathrm{U}(n)$ [27] and are left with

$$
\begin{aligned}
\mathcal{I}= & \int d \alpha_{11} d \alpha_{11}^{*} \cdots d \alpha_{m n} d \alpha_{m n}^{*} \mathcal{T}_{m, n}\left(\alpha_{11}, \ldots, \alpha_{m n}, \alpha_{11}^{*}, \ldots, \alpha_{m n}^{*}\right) \\
& \times\left(\frac{\operatorname{det}\left(\lambda_{j}^{m-i} I_{m-i}\left(\lambda_{j}\right)\right)_{i, j=1, \ldots, m}}{\Delta\left(\lambda_{1}^{2}, \ldots, \lambda_{m}^{2}\right)}\right)\left(\frac{\operatorname{det}\left(\mu_{j}^{n-i} I_{n-i}\left(\mu_{j}\right)\right)_{i, j=1, \ldots, n}}{\Delta\left(\mu_{1}^{2}, \ldots, \mu_{n}^{2}\right)}\right),
\end{aligned}
$$

where $\lambda_{1}^{2}, \ldots, \lambda_{m}^{2}$ are the eigenvalues of the matrix $\tilde{A}_{m} \tilde{B}_{m}, \mu_{1}^{2}, \ldots, \mu_{n}^{2}$ are the eigenvalues of the matrix $\tilde{A}_{n} \tilde{B}_{n}$, and $I_{\nu}$ is the modified Bessel function of the first kind. Furthermore, $\Delta\left(\lambda_{1}^{2}, \ldots, \lambda_{m}^{2}\right) \equiv \prod_{1 \leq i<j \leq m}\left(\lambda_{i}^{2}-\lambda_{j}^{2}\right)$ is the Vandermonde determinant.

In the case of $\mathrm{U}(1 \mid 1)$ this is equal to

$$
\mathcal{I}=\int d \alpha_{11}^{*} d \alpha_{11} I_{0}\left(\left[a_{1} b_{1}\left(1+\alpha_{11}^{*} \alpha_{11}\right)\right]^{1 / 2}\right) I_{0}\left(\left[a_{2} b_{2}\left(1-\alpha_{11}^{*} \alpha_{11}\right)\right]^{1 / 2}\right) .
$$

We expand the Bessel functions in order to obtain the linear term in $\alpha_{11}^{*} \alpha_{11}$,

$$
\begin{aligned}
& I_{0}\left(\left[a_{1} b_{1}\left(1+\alpha_{11}^{*} \alpha_{11}\right)\right]^{1 / 2}\right)=\sum_{k=0}^{\infty} \frac{1}{(k !)^{2}}\left(\frac{a_{1} b_{1}}{4}\right)^{k}\left(1+k \alpha_{11}^{*} \alpha_{11}\right) \\
& I_{0}\left(\left[a_{2} b_{2}\left(1-\alpha_{11}^{*} \alpha_{11}\right)\right]^{1 / 2}\right)=\sum_{\ell=0}^{\infty} \frac{1}{(\ell !)^{2}}\left(\frac{a_{2} b_{2}}{4}\right)^{\ell}\left(1-\ell \alpha_{11}^{*} \alpha_{11}\right) .
\end{aligned}
$$

Thus, we can write

$$
\begin{aligned}
\mathcal{I} & =\frac{1}{2}\left(\sqrt{a_{1} b_{1}} \frac{\partial}{\partial \sqrt{a_{1} b_{1}}}-\sqrt{a_{2} b_{2}} \frac{\partial}{\partial \sqrt{a_{2} b_{2}}}\right) I_{0}\left(\sqrt{a_{1} b_{1}}\right) I_{0}\left(\sqrt{a_{2} b_{2}}\right) \\
& =\frac{1}{2} \operatorname{det}\left(\begin{array}{ll}
I_{0}\left(\sqrt{a_{1} b_{1}}\right) & \sqrt{a_{1} b_{1}} I_{1}\left(\sqrt{a_{1} b_{1}}\right) \\
I_{0}\left(\sqrt{a_{2} b_{2}}\right) & \sqrt{a_{2} b_{2}} I_{1}\left(\sqrt{a_{2} b_{2}}\right)
\end{array}\right) .
\end{aligned}
$$

This is equivalent to the special case of our general result given in Eq. (2). 
Let us now turn to the case of $\mathrm{U}(2 \mid 1)$, which is slightly more involved. In this case the calculation of the eigenvalues yields

$$
\begin{aligned}
& \lambda_{1}^{2}=a_{1} b_{1}\left(1-\alpha_{11} \alpha_{11}^{*}-\frac{a_{1} b_{1}+2 a_{2} b_{2}}{3\left(a_{1} b_{1}-a_{2} b_{2}\right)} \alpha_{11} \alpha_{11}^{*} \alpha_{21} \alpha_{21}^{*}\right) \\
& \lambda_{2}^{2}=a_{2} b_{2}\left(1-\alpha_{21} \alpha_{21}^{*}+\frac{2 a_{1} b_{1}+a_{2} b_{2}}{3\left(a_{1} b_{1}-a_{2} b_{2}\right)} \alpha_{11} \alpha_{11}^{*} \alpha_{21} \alpha_{21}^{*}\right), \\
& \mu_{1}^{2}=a_{3} b_{3}\left(1+\alpha_{11} \alpha_{11}^{*}+\alpha_{21} \alpha_{21}^{*}+\frac{2}{3} \alpha_{11} \alpha_{11}^{*} \alpha_{21} \alpha_{21}^{*}\right) .
\end{aligned}
$$

The calculation of the determinants of Bessel functions of these eigenvalues is tedious but straightforward. Finally, we find that the term proportional to $\alpha_{11} \alpha_{11}^{*} \alpha_{21} \alpha_{21}^{*}$ is indeed

$$
\frac{1}{a_{1} b_{1}-a_{2} b_{2}} \operatorname{det}\left(\begin{array}{ccc}
I_{0}\left(\sqrt{a_{1} b_{1}}\right) & I_{0}\left(\sqrt{a_{2} b_{2}}\right) & I_{0}\left(\sqrt{a_{3} b_{3}}\right) \\
\sqrt{a_{1} b_{1}} I_{1}\left(\sqrt{a_{1} b_{1}}\right) & \sqrt{a_{2} b_{2}} I_{1}\left(\sqrt{a_{2} b_{2}}\right) & \sqrt{a_{3} b_{3}} I_{1}\left(\sqrt{a_{3} b_{3}}\right) \\
a_{1} b_{1} I_{2}\left(\sqrt{a_{1} b_{1}}\right) & a_{2} b_{2} I_{2}\left(\sqrt{a_{2} b_{2}}\right) & a_{3} b_{3} I_{2}\left(\sqrt{a_{3} b_{3}}\right)
\end{array}\right) .
$$

This is again equivalent to the special case of our general result given in Eq. (2). Note that this result also proves the power series identity conjectured in App. $\mathrm{D}$ for $m=2, n=1$. We do not present the calculation for $m=1, n=2$ here since it is very similar to the case of $m=2, n=1$. The next step would be to perform this procedure for general $\mathrm{U}(m \mid n)$, which would then also yield a proof of the conjecture for arbitrary values of $m$ and $n$.

[1] K. B. Efetov, Supersymmetry in Disorder and Chaos (Cambridge University Press, 1997).

[2] E. Abdalla, A. Zadra, M. C. B. Abdalla, and D. Dalmazi, Lect. Notes Phys. M20, 1 (1994).

[3] M. L. Mehta, Random matrices (Elsevier/Academic Press, Amsterdam, 2004), 3rd ed.

[4] T. Guhr, A. Müller-Groeling, and H. A. Weidenmüller, Phys. Rept. 299, 189 (1998), cond-mat/9707301.

[5] J. J. M. Verbaarschot and T. Wettig, Ann. Rev. Nucl. Part. Sci. 50, 343 (2000), hep-ph/0003017.

[6] S. Helgason, Groups and Geometric Analysis (Academic Press, San Diego, 1984).

[7] S. Helgason, Differential Geometry, Lie Groups, and Symmetric Spaces (Oxford University Press, 2001).

[8] L. K. Hua, Harmonic Analysis of Functions of Several Complex Variables in the Classical Domains (AMS, Providence, R.I., 1963).

[9] Harish-Chandra, Am. J. Math. 79, 87 (1957).

[10] C. Itzykson and J. B. Zuber, J. Math. Phys. 21, 411 (1980).

[11] T. Guhr and H. Kohler, J. Math. Phys. 43, 2707 (2002), math-ph/0011007.

[12] F. A. Berezin and F. I. Karpelevich, Dokl. Akad. NAUK (SSSR) 118, 9 (1958).

[13] T. Guhr and T. Wettig, J. Math. Phys. 37, 6395 (1996), hep-th/9605110.

[14] A. D. Jackson, M. K. Sener, and J. J. M. Verbaarschot, Phys. Lett. B387, 355 (1996), hep-th/9605183.

[15] H. Leutwyler and A. Smilga, Phys. Rev. D46, 5607 (1992).

[16] F. A. Berezin, Introduction to Superanalysis (AA Dordrecht, Holland, 1987).

[17] K. B. Efetov, Adv. Phys. 32, 53 (1983).

[18] J. J. M. Verbaarschot and M. R. Zirnbauer, J. Phys. A18, 1093 (1985).

[19] J. J. M. Verbaarschot, H. A. Weidenmüller, and M. R. Zirnbauer, Phys. Rept. 129, 367 (1985).

[20] T. Guhr, J. Math. Phys. 32, 336 (1991).

[21] A. D. Jackson, M. K. Sener, and J. J. M. Verbaarschot, Nucl. Phys. B506, 612 (1997), hep-th/9704056.

[22] T. Guhr and H. Kohler, J. Math. Phys. 45, 3636 (2004), math-ph/0212060.

[23] V. Serganova, private communication (1992).

[24] M. R. Zirnbauer, J. Phys. A 29, 7113 (1996).

[25] A. B. Balantekin, Phys. Rev. D62, 085017 (2000), hep-th/0007161.

[26] A. B. Balantekin, Phys. Rev. E64, 066105 (2001), cond-mat/0109112.

[27] B. Schlittgen and T. Wettig, J. Phys. A36, 3195 (2003), math-ph/0209030.

[28] A. B. Balantekin and P. Cassak, J. Math. Phys. 43, 604 (2002), hep-th/0108130.

[29] S. L. Shatashvili, Commun. Math. Phys. 154, 421 (1993), hep-th/9209083.

[30] I. M. Gelfand and M. L. Tzetlin, Dokl. Akad. Nauk 71, 825 (1950).

[31] T. Guhr, Comm. Math. Phys. 176, 555 (1996).

[32] T. Guhr and H. Kohler, J. Math. Phys. 43, 2741 (2002), math-ph/0012047.

[33] J. Gasser and H. Leutwyler, Phys. Lett. B188, 477 (1987).

[34] A. B. Balantekin, J. Math. Phys. 25, 2028 (1984).

[35] A. B. Balantekin and I. Bars, J. Math. Phys. 22, 1149 (1981). 
[36] A. B. Balantekin and I. Bars, J. Math. Phys. 22, 1810 (1981).

[37] J. Alfaro, R. Medina, and L. F. Urrutia, J. Math. Phys. 38, 5319 (1997), hep-th/9702112.

[38] H. Weyl, The Classical Groups (Princeton Univ. Press, Princeton, N.J., 1946).

[39] M. Abramowitz and A. Stegun, Pocketbook of Mathematical Functions (Harri Deutsch, 1984).

[40] T. Akuzawa and M. Wadati, J. Phys. Soc. Jap. 67, 2151 (1998), hep-th/9804049.

[41] http://gmplib.org.

[42] I. G. Macdonald, Symmetric Functions and Hall Polynomials (Clarendon, Oxford, 1979).

[43] T. Guhr, J. Math. Phys. 34, 2523 (1993).

[44] T. Guhr, J. Math. Phys. 34, 2541 (1993).

[45] P. H. Damgaard, J. C. Osborn, D. Toublan, and J. J. M. Verbaarschot, Nucl. Phys. B547, 305 (1999), hep-th/9811212. 